\title{
TRANSFORMATION OF A GRID OF QUADRATS TO COPE WITH PERSPECTIVE ARTIFACTS
}

\author{
Luis M. CRUZ-ORIVE凶 AND MARCOS CRUZ \\ Department of Mathematics, Statistics and Computation, Faculty of Sciences, University of Cantabria, Avda. \\ Los Castros 48, E-39005 Santander, Spain. \\ e-mail: luis.cruz@unican.es, marcos.cruz@unican.es \\ (Received October 22, 2019; accepted February 17, 2020)
}

\begin{abstract}
The direct superimposition of a standard test grid of congruent quadrats onto an image bearing a population of particles exhibiting perspective artifacts, tends to increase the variance of the population size estimator, because the quadrat contents become unbalanced. If the quadrats are transformed according to the same projection mechanism affecting the particles, however, then the variance is restored into moderate values. Our purpose was to provide exact, easily programmable equations for the relevant transform.
\end{abstract}

Keywords: cylindrical projection, gigapixel image, particle number, quadrat grid, sinusoidal curve, transformed quadrat grid.

Dedicated to the memory of Ewald R. Weibel.

\section{INTRODUCTION}

This note was motivated by the problem of estimating the finite size of a fixed and bounded population of objects in a reference plane. An object is regarded as a 'particle', namely a compact and connected set separated from other particles. In practice the particles (people, animals, or whatever distinguishable objects) are observable in a reference projection plane, henceforth called the horizontal plane (HP), and observed in a flat image - usually a photograph - called the image plane (IP). Ideally, the IP should be parallel - at least approximately - to the HP, in order to avoid perspective artifacts. Cruz et al. (2015) proposed to superimpose on the IP a uniform random grid of quadrats, with a fundamental square tile of area $a>0$, containing a fundamental square quadrat of area $a^{\prime} \in(0, a]$, whereby the sampling period is $a / a^{\prime} \geq 1$ and

$$
\widehat{N}=\frac{a}{a^{\prime}} \cdot Q,
$$

is an unbiased estimator (UE) of the population size $N$. Here $Q$ is the sample size, namely the total number of particles captured by the quadrats. For general particles, $Q$ is scored using the forbidden line rule of Gundersen (1977), widely used in stereology to cope with edge effects. The free software CountEm (https://countem.unican.es/) offers a computer assisted procedure to estimate $N$ on real images. For further details on the sampling design see Gómez et al. (2019).
For large populations (e.g., for $N \gtrsim 10,000$ ), panoramic 'gigapixel' images are nowadays obtained with the aid of special cameras which use cylindrical projections from some elevated observation point which plays the role of a centre of projection. The resulting images exhibit perspective artifacts: the observed particle density increases with the distance from the observation point. As a consequence, distant quadrats will tend to capture many more particles than proximal quadrats, this causing $\operatorname{Var}(\widehat{N})$ to become unduly large (Cruz and González-Villa, 2019). An idea proposed in the latter paper was to apply to the quadrats the same projection mechanism used for the gigapixel image. Now, for a given position of the grid the individual quadrat counts will be identical to those obtained with an ordinary grid on an IP parallel to the HP, whereby the corresponding estimation variance will be restored. Each transformed quadrat was drawn approximately by applying the pertinent point transform to each of a few landmarks in the quadrat boundary, then joining the transformed landmarks. In this note we give explicit projection formulae to plot the transformed grid of quadrats directly on the IP. This is done by computing first the cylindrical projection of an ordinary grid from a fixed point, and then unrolling the cylinder. The edges of the transformed quadrats turn out to be sinusoidal arcs.

\section{CYLINDRICAL PROJECTIONS}

\section{SETUP}

A special camera for gigapixel images consists of a lens which can rotate around a vertical axis; at each 
position, the camera collects a partial, essentially flat image, and a suitable software creates a paving of such images which can map the target object into a panoramic view of up to $360^{\circ}$. The result is roughly equivalent to projecting the object through a pinhole onto the internal face of a right circular cylinder. Based on this fact, the model described below involves projection of points and lines in the HP through a centre of projection onto a vertical right circular cylinder. For clarity of exposition the projections are made onto the external surface of the cylinder, but the results are equally valid for internal projections.

Fix an orthogonal reference frame $o x_{1} x_{2} x_{2}$ in $\mathbb{R}^{3}$. We assume that the $N$ particles of the target population are mapped 1:1 into a bounded set of $N$ associated points $\left\{y_{1}, y_{2}, \ldots, y_{N}\right\}$ contained in a horizontal plane (HP) $x_{3}=h$, where $h$ is a constant. The origin $o$ is adopted as the centre of projection. The HP will usually lie below $o$, hence to visualize the setup we may assume that $h<0$, see Fig. 1a. Consider a vertical, right circular cylinder of radius $r>0$, namely,

$$
C=\left\{\left(x_{1}, x_{2}, x_{3}\right): x_{1}^{2}+x_{2}^{2}=r^{2}\right\} .
$$

A cylindrical projection of a point $y$ in the HP is the unique intersection point $y^{\prime}$ determined in $C$ by a ray emanating from $o$ through $y$. Let $O$ denote the point $(r, 0,0)$. When the cylinder is unrolled we obtain the image plane (IP). In the IP, the image of the circle

$$
C_{0}=\left\{\left(x_{1}, x_{2}, 0\right): x_{1}^{2}+x_{2}^{2}=r^{2}\right\}
$$

is a straight line segment of length $2 \pi r$, whose support line is adopted as the axis of abscissas $O X_{1}$ of a rectangular frame $O X_{1} X_{2}$. Given a point, or a straight line, in the HP, we want to represent it in the IP.

\section{CYLINDRICAL PROJECTION OF A POINT}

Let $(R, \omega, h), R \in(0, \infty), \omega \in[0,2 \pi), h \in \mathbb{R}$, denote the cylindrical coordinates of a point $y$ in the HP, see Fig. 1a. The cylindrical coordinates of the corresponding cylindrical projection $y^{\prime}$ are $(r, \omega, h r / R)$, and therefore the Cartesian coordinates of the point $y^{\prime}$ in the IP are,

$$
\left\{\begin{array}{l}
X_{1}=r \omega, \\
X_{2}=h r / R,
\end{array}\right.
$$

see Fig. 1b. In this way, the target set of $N$ associated points $\left\{y_{1}, y_{2}, \ldots, y_{N}\right\}$ is mapped into the set of $N$ points $\left\{\left(X_{1 i}, X_{2 i}\right), i=1,2, \ldots, N\right\}$ in the IP, which constitute the relevant image data. Note that these data are generally unknown - only a small sample, acquired with a test system of quadrats, will be available to estimate $N$.

\section{CYLINDRICAL PROJECTION OF A STRAIGHT LINE}

Consider a straight line of normal coordinates $p>0, \phi \in[0,2 \pi)$ contained in the HP, namely,

$$
L_{1}=\left\{\left(x_{1}, x_{2}, h\right): x_{1} \cos \phi+x_{2} \sin \phi-p=0\right\} .
$$

A cylindrical projection from $o$ maps the straight line into the ellipse $C \cap L_{2}$, see Fig. 1c, where $L_{2}$ denotes the plane containing $o$ and the straight line $L_{1}$, namely,

$$
L_{2}=\left\{\left(x_{1}, x_{2}, x_{3}\right): h x_{1} \cos \phi+h x_{2} \sin \phi-p x_{3}=0\right\} .
$$

Set $\varphi=\phi-\pi / 2$, and let $\alpha \in[0,2 \pi)$ be the polar angle measured in the horizontal reference plane $o x_{1} x_{2}$ with origin at an axis which makes an angle $\varphi$ with the $o x_{1}$ axis, (namely with an axis parallell to $L_{1}$ through $o$ ). The parametric equations of the ellipse $C \cap L_{2}$ in terms of $\varphi$ and $\alpha$ are the following,

$$
\left\{\begin{array}{l}
x_{1}=r \cos (\varphi+\alpha), \\
x_{2}=r \sin (\varphi+\alpha), \\
x_{3}=(h r / p) \sin \alpha .
\end{array}\right.
$$

The preceding expression of $x_{3}$ is found by substituting the expressions of $x_{1}, x_{2}$ into the equation of the plane $L_{2}$ containing the ellipse. Note that $x_{3}$ does not depend on $\varphi$. Thus, the parametric equations of the ellipse map $C \cap L_{2}$ in the IP become

$$
\left\{\begin{array}{l}
X_{1}=r(\varphi+\alpha), \\
X_{2}=(h r / p) \sin \alpha,
\end{array}\right.
$$

or, in Cartesian coordinates,

$$
X_{2}=\frac{h r}{p} \sin \left(\frac{X_{1}}{r}-\varphi\right)
$$

which corresponds to a sinusoidal curve, as expected, see e.g. Steinhaus (1950), Ch.10, and Fig. 1d.

\section{CYLINDRICAL PROJECTION OF A SQUARE GRID OF QUADRATS}

For convenience we define a square grid of square quadrats in the HP as follows. A stripe $L_{t}(p, \phi)$ of thickness $t>0$ is the portion of the plane comprised between two parallel straight lines of normal coordinates $(p, \phi)$ and $(p+t, \phi)$, respectively. A systematic series of stripes (called Cavalieri stripes in stereology) of fixed period $T \geq t$ is

$$
\left\{L_{t}(z+k T, \phi), k \in \mathbb{Z}\right\},
$$




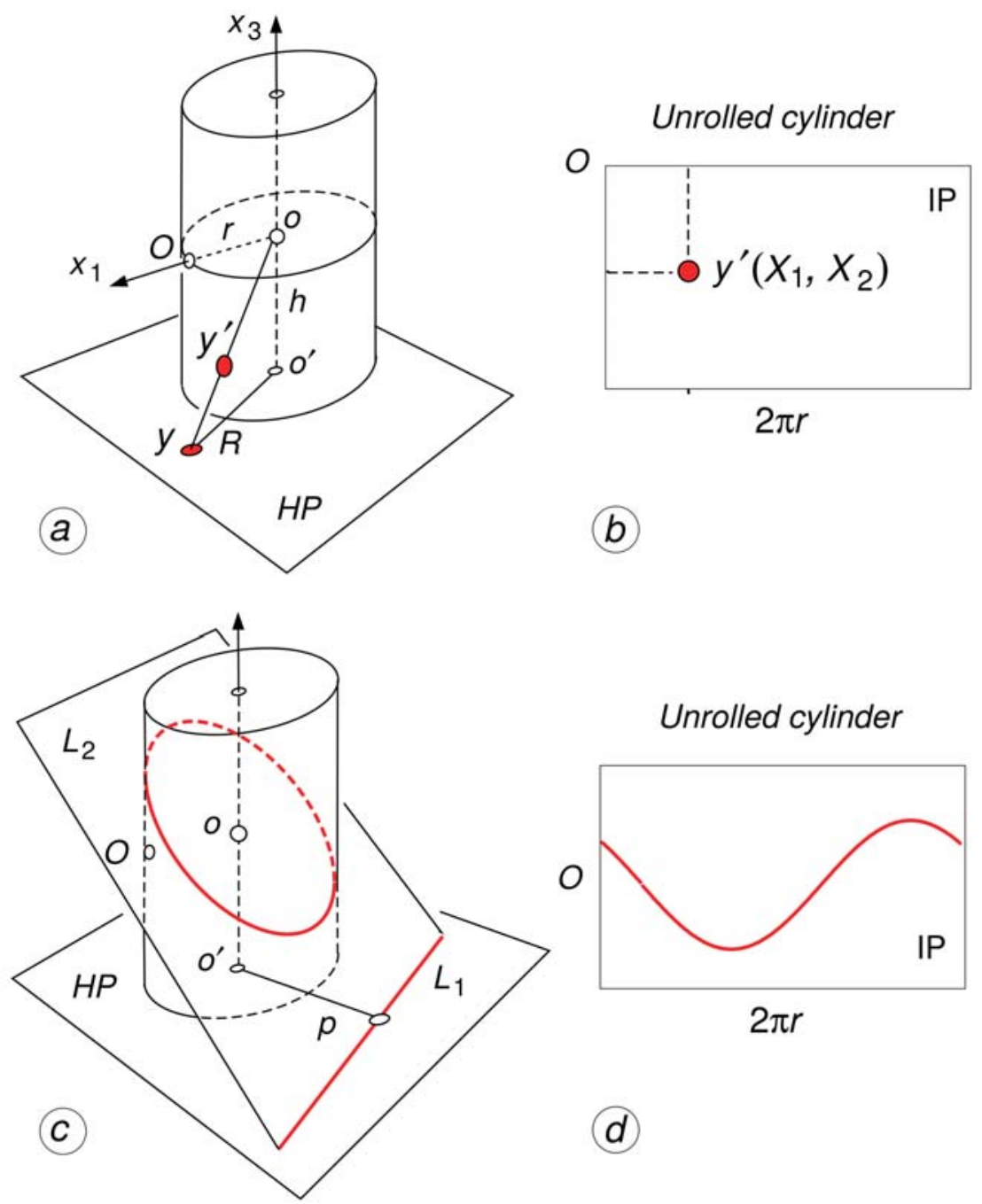

Fig. 1. (a) Cylindrical projection of a point $y$ in the horizontal plane, onto the point $y^{\prime}$, with projection centre $o$. (b) Cartesian coordinates of $y^{\prime}$ in the image plane, see Eq. 4. (c) Cylindrical projection of a straight line in the horizontal plane, into an ellipse. (d) In the image plane, the transform of the ellipse is a sinusoidal curve, see Eq. 9.

where $z \sim \operatorname{UR}[0, T)$ is a uniform random offset in the interval $[0, T)$, see Fig. 2a. Then, the intersection between two mutually perpendicular Cavalieri stripe series with independent UR offsets $z_{1}, z_{2}$ in $[0, T)$, namely,

$$
\begin{array}{r}
\Lambda_{t, T}(\phi)=\left\{L_{t}\left(z_{1}+j T, \phi\right)\right\} \cap\left\{L_{t}\left(z_{2}+k T, \phi+\pi / 2\right),\right. \\
j, k \in \mathbb{Z}\}, \phi \in[0, \pi / 2),
\end{array}
$$

is a square grid of square quadrats of side length $t$ and gap length $T-t \geq 0$, see Fig. $2 b$.

By virtue of Eq. 9, the transform of a stripe $\left\{L_{t}(z+k T, \phi)\right\}$ in the IP is the portion of space comprised between two sinusoidal curves of equations

$$
\left\{\begin{array}{l}
X_{2}=\frac{h r}{z+k T} \cdot \sin \left(\frac{X_{1}}{r}-\varphi\right) \\
X_{2}=\frac{h r}{z+k T+t} \cdot \sin \left(\frac{X_{1}}{r}-\varphi\right)
\end{array}\right.
$$

respectively. For the transform of the perpendicular stripe $L_{t}(z+j T, \phi+\pi / 2)$ the equations are analogous to the preceding ones with $\sin (\cdot)$ replaced with $\cos (\cdot)$. With this information it is easy to write a software routine to plot the transform of the test system $\Lambda_{t, T}(\phi)$ in the IP, see Fig. 2b,c. Note that a change in the orientation $\phi$ of the original test system causes only a shift of the transformed test system along the horizontal axis $O X_{1}$ in the IP. 


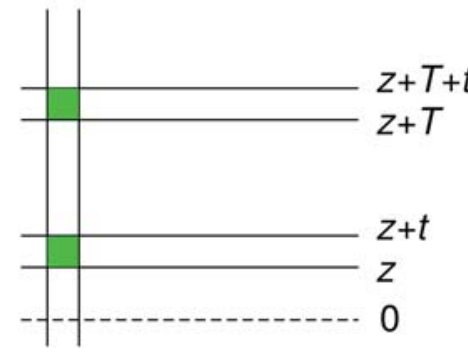

(a)

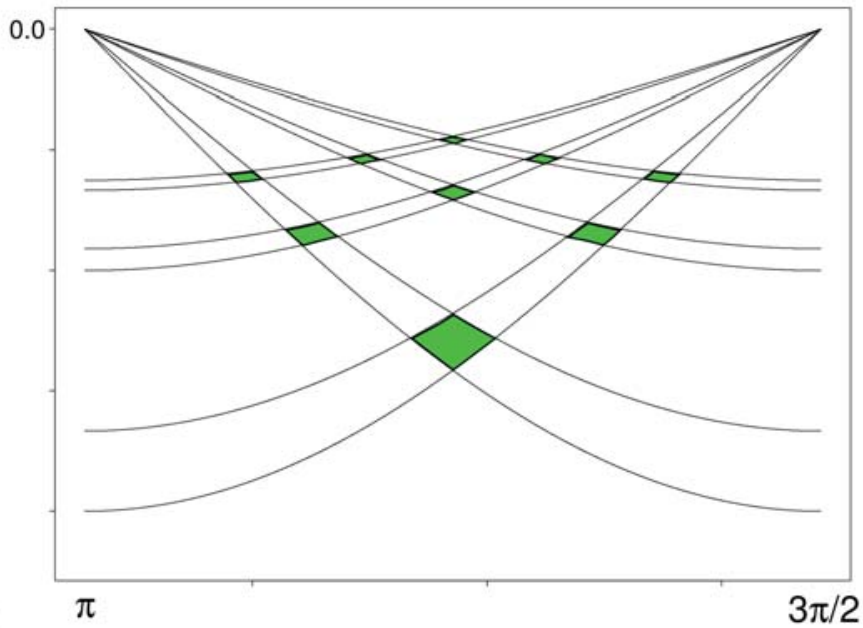

Fig. 2. (a) Quadrat generation as the intersection of two mutually perpendicular, uniform random and independent systems of Cavalieri stripes. (b) A portion of a square test system of square quadrats in the horizontal plane. The quadrats bear a portion of the forbidden edge (in red) to apply the corresponding unbiased counting rule. (c) Generation of the transformed test system in the image plane by means of the intersection of two families of sinusoids with $\varphi=0$ and $\varphi=p i / 2$, respectively, see Eq. 12. The transformed quadrats should also bear the corresponding forbidden lines, which are portions of sinusoidal curves (see Fig. 3c).

\section{A NOTE ON THE PERSPECTIVE EFFECT}

Consider two points $y_{1}, y_{2}$ in the HP, of cylindrical coordinates $(R, \omega, h)$ and $(R+\delta, \omega, h)$ respectively. Thus, for simplicity the two points are collinear with the point $o^{\prime}(0,0, h)$, with a distance $\delta>0$ between them. By the second Eq. 4, the transforms of $y_{1}, y_{2}$ in the IP lie in a vertical line, and have ordinates $X_{21}=h r / R$ and $X_{22}=h r /(R+\delta)$, respectively. Thus, the apparent distance between the two point transforms in the IP is,

$$
\begin{aligned}
X_{22}-X_{21} & =h r\left(\frac{1}{R+\delta}-\frac{1}{R}\right) \\
& =\frac{-h r \delta}{R(R+\delta)}=O\left(\frac{1}{R^{2}}\right),
\end{aligned}
$$

which shows that the perspective effect is of order $R^{-2}$.

\section{SYNTHETIC EXAMPLE}

To illustrate the preceding theory we generated in the HP a realization of $N=300$ point particles within a rectangle from a hard core point process, with the aid of the package Spatstat from the statistical software R (https://www.r-ptoject.org/), see Fig. 3a. Here the HP played the role of a preliminary IP, with origin at the vertical projection $o^{\prime}(0,0, h=-2)$ of the centre of projection $o(0,0,0)$, see Fig. 1a. With this setup, the containing rectangle was $[-5,15) \times[2,12)$. To estimate the target number $N$, a grid was superimposed on the IP, consisting of square quadrats of side length $t=1$, with a square fundamental tile of side length $T=4$. The sampling period was therefore $a / a^{\prime}=$ 16. In Fig. 3a, the observed sample size, namely the total number of particle centres within the quadrats, 


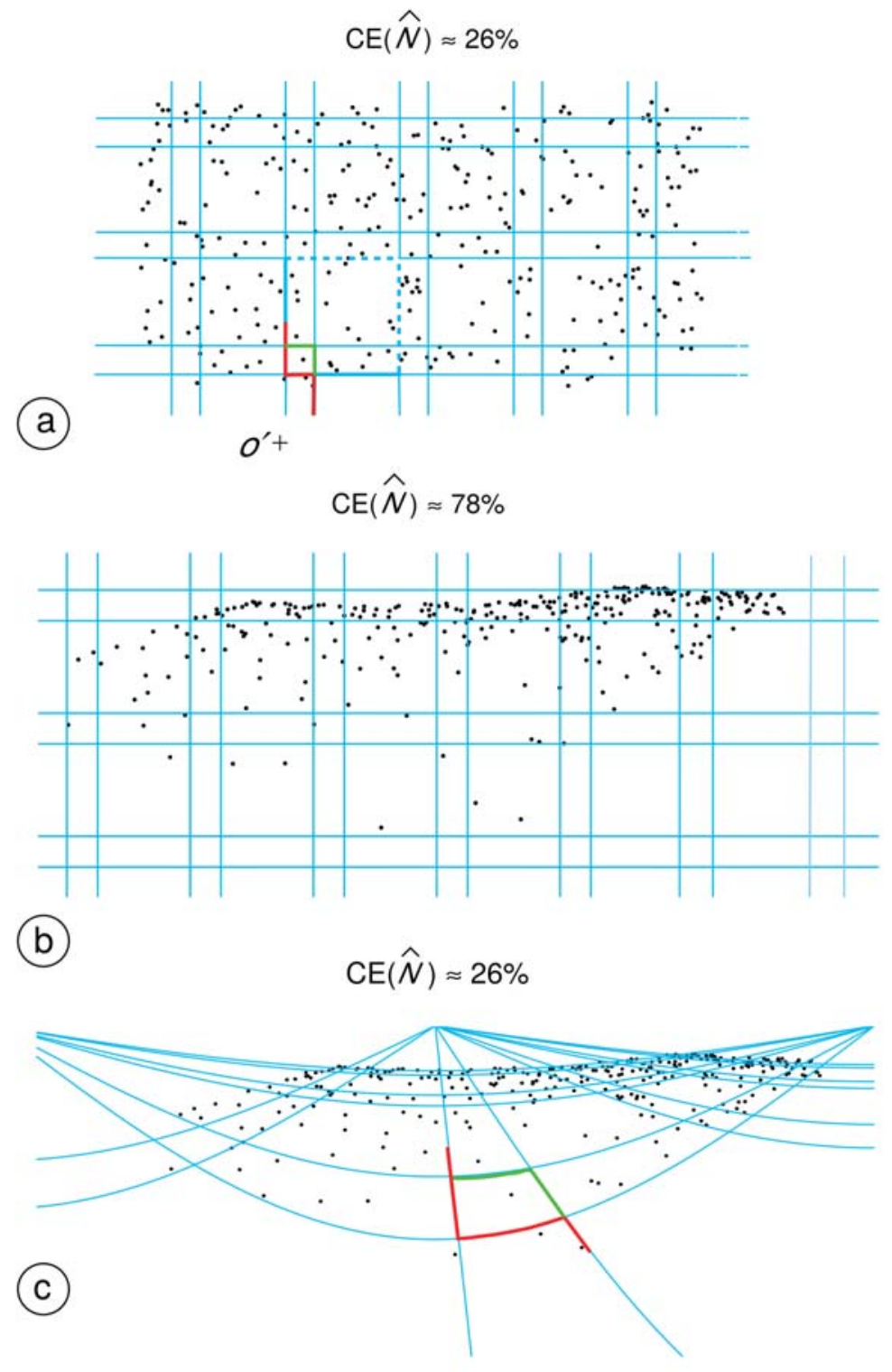

Fig. 3. Illustration of the Section Synthetic example. (a) A realization of $N=300$ particle points in a rectangle, from a hardcore point process. A quadrat grid is superimposed with which the empirical coefficient of error of the number estimator is as given (26\%). A quadrat is equipped with a portion of the forbidden edges (not required in this study), mainly to compare it with its transform in Fig. 3c. (b) After cylindrical projection and posterior unrolling, the resulting particle point pattern is rather inhomogeneous. Unbiasedness is preserved if the grid is uniform random, but the square coefficient of error increases by a factor of about 9. (c) If the ordinary grid is replaced with the one given by Eq. 12, which uses the same transformation which maps the pattern in (a) into the one in (b), then the individual quadrat counts are identical to those in (a), and consequently the estimation precision is restored.

is $Q=20$, whereby Eq. 1 yields $\widehat{N}=320$, with a relative deviation of $6.7 \%$. The empirical coefficient of error $\mathrm{CE}_{\mathrm{e}}(\widehat{N})=\mathrm{SD}_{\mathrm{e}}(\widehat{N}) / N \approx 0.26$ was computed from 1024 automatic Monte Carlo superimpositions of the grid using a systematic resampling design (usually more efficient than independent resampling) - for details see Cruz et al. (2015). This $\mathrm{CE}_{\mathrm{e}}(\widehat{N})$ was larger than usual due to the simplicity of the example - in practice the recommended sample size $Q$ to attain a reasonable precision ranges from about 100 to 200 (Cruz and González-Villa, 2018).

The point particles of Fig. 3a were projected 
through $o$ onto a vertical circular cylinder of radius $r=1.4$. After unrolling the cylinder, the transformed IP is represented in Fig. 3b. The superimposition of the (properly scaled) grid of quadrats described above, with the same sampling period of 16 , now yielded $\mathrm{CE}_{\mathrm{e}}(\widehat{N}) \approx 0.78$. Thus, the estimation of $\widehat{N}$ is in this case about $(78 / 26)^{2}=9$ times less efficient than in the case illustrated in Fig. 3a. The purpose of this study was to revert the impact of the transform operation induced by the cylindrical projection of the particles by means of a square grid of square quadrats submitted to the same transformation. With the given parameters, and with $\varphi=0$ and $\varphi=\pi / 2$, the relevant Eq. 12 yielded the grid transform illustrated in Fig. 3c. For comparison, two corresponding quadrats from Fig. 3a and Fig. 3c, respectively, are displayed with portions of the forbidden edge (in red) associated with them. It can be readily checked that the individual particle counts are identical for all pairs of corresponding quadrats. In other words, the data collected in Fig. 3a and Fig. 3c, with $Q=20$ in particular, are identical, and therefore the initial $\mathrm{CE}_{\mathrm{e}}(\widehat{N}) \approx 0.26$ will also hold for Fig. 3c without any further qualification.

\section{DISCUSSION}

Cruz and González-Villa (2019) implemented the design illustrated in Fig. $3 \mathrm{c}$ to estimate the size of a large demonstration from high resolution gigapixel images. Proper computer assisted zooming facilitated individual counting in distant quadrats. In that paper, however, the shape of the transformed quadrats was computed numerically from point landmarks, which affected the efficiency of the procedure. This fact prompted us to treat the model mathematically, showing that the transformed quadrats are bounded by arcs of sinusoidal curves, whose automatic plotting is straightforward.

The projection cylinder used in our model does not have a physical counterpart - it is just a convenient model. Its radius $r$, however, is involved in Eq. 12, which determines the transformed grid. On the other hand, the coordinates of the projection centre, and $h$ in particular, do have a real counterpart because they determine the position of the camera, which is usually not directly available either. In the aforementioned paper, the estimation of the auxiliary parameter $r$, and of the camera coordinates, were estimated indirectly from a system of equations involving the coordinates (obtained with the aid of Google Earth) of physical landmarks available in the scenario of the demonstration. This suggests that the implementation of the design described here on a given gigapixel image may not be that straightforward at present in the absence of the additional information required.

\section{ACKNOWLEDGEMENT}

A preliminary version of this paper was read at the Workshop on Image Analysis and Stereology with applications in Biology and Social Sciences (WIASABiSS), E-Santander, September 11-14, 2018.

\section{REFERENCES}

Cruz M, Gómez D, Cruz-Orive LM (2015). Efficient and unbiased estimation of population size. PLoS ONE 10(11):e0141868.

Cruz M, González-Villa J (2018). Simplified procedure for efficient and unbiased population size estimation. PLoS ONE 13(10):e0206091.

Cruz M, González-Villa J (2019). Unbiased population size estimation on still gigapixel images. Sociol Method Res 0049124118799373.

Gómez A, Cruz M, Cruz-Orive LM (2019). Variance prediction for population size estimation. Image Anal Stereol 38:131-9.

Gundersen HJG (1977). Notes on the estimation of the numerical density of arbitrary profiles: the edge effect. J Microsc 111:219-23.

Steinhaus H (1950). Mathematical snapshots. New York: Oxford University Press. 